\title{
Heralded source of bright multi-mode mesoscopic sub-Poissonian light
}

\author{
Iskhakov, Timur; Usenko, V. C.; Andersen, Ulrik Lund; Filip, R.; Chekhova, M. V.; Leuchs, G.
}

Published in:

Optics Letters

Link to article, DOI:

10.1364/OL.41.002149

Publication date:

2016

Document Version

Peer reviewed version

Link back to DTU Orbit

Citation $(A P A)$ :

Iskhakov, T., Usenko, V. C., Andersen, U. L., Filip, R., Chekhova, M. V., \& Leuchs, G. (2016). Heralded source of bright multi-mode mesoscopic sub-Poissonian light. Optics Letters, 41(10), 2149-2152.

https://doi.org/10.1364/OL.41.002149

\section{General rights}

Copyright and moral rights for the publications made accessible in the public portal are retained by the authors and/or other copyright owners and it is a condition of accessing publications that users recognise and abide by the legal requirements associated with these rights.

- Users may download and print one copy of any publication from the public portal for the purpose of private study or research.

- You may not further distribute the material or use it for any profit-making activity or commercial gain

- You may freely distribute the URL identifying the publication in the public portal

If you believe that this document breaches copyright please contact us providing details, and we will remove access to the work immediately and investigate your claim. 


\section{Heralded source of bright multi-mode mesoscopic sub-Poissonian light}

Iskhakov, Timur; Usenko, V. C.; Andersen, Ulrik Lund; Filip, R.; Chekhova, M. V.; Leuchs, G.

Published in:

Optics Letters

DOI:

10.1364/OL.41.002149

Publication date:

2016

Document Version

Publisher's PDF, also known as Version of record

Link to publication

Citation (APA):

Iskhakov, T., Usenko, V. C., Andersen, U. L., Filip, R., Chekhova, M. V., \& Leuchs, G. (2016). Heralded source of bright multi-mode mesoscopic sub-Poissonian light. Optics Letters, 41(10), 2149-2152. DOI:

10.1364/OL.41.002149

- You may freely distribute the URL identifying the publication in the public portal ? 


\title{
Heralded source of bright multi-mode mesoscopic sub-Poissonian light
}

\author{
T. Sh. Iskhakov, ${ }^{1,2, *}$ V. C. Usenko, ${ }^{3}$ U. L. Andersen, ${ }^{1,2}$ R. Filip, ${ }^{3}$ M. V. Chekhova, ${ }^{2,4,5}$ and G. Leuchs ${ }^{2,4}$ \\ ${ }^{1}$ Department of Physics, Technical University of Denmark, Fysikvej Building 309, Kgs. Lyngby 2800, Denmark \\ ${ }^{2}$ Max-Planck Institute for the Science of Light, Guenther-Scharowsky-Str. 1 / Bau 24, Erlangen D-91058, Germany \\ ${ }^{3}$ Department of Optics, Palacky University, 17 Listopadu 12, Olomouc 771 46, Czech Republic \\ ${ }^{4}$ University of Erlangen-Nürnberg, Staudtstrasse 7/B2, Erlangen 91058, Germany \\ ${ }^{5}$ Physics Department, Moscow State University, Leninskiye Gory 1-2, Moscow 119991, Russia \\ ${ }^{*}$ Corresponding author: tiskha@gmail.com
}

Received 8 March 2016; revised 4 April 2016; accepted 5 April 2016; posted 8 April 2016 (Doc. ID 260767); published 3 May 2016

\begin{abstract}
In a direct detection scheme, we observed $7.8 \mathrm{~dB}$ of twinbeam squeezing for multi-mode two-color squeezed vacuum generated via parametric downconversion. Applying postselection, we conditionally prepared a sub-Poissonian state of light containing $6.3 \cdot 10^{5}$ photons per pulse on the average with the Fano factor $0.63 \pm 0.01$. The scheme can be considered as the heralded preparation of pulses with the mean energy varying between tens and hundreds of $f J$ and the uncertainty considerably below the shot-noise level. Such pulses can be used in metrology (for instance, for radiometer calibration), as well as for probing multi-mode nonlinear optical effects. () 2016 Optical Society of America
\end{abstract}

OCIS codes: (270.0270) Quantum optics; (270.6570) Squeezed states; (270.5290) Photon statistics.

http://dx.doi.org/10.1364/OL.41.002149

Quantum optics provides the recipes to overcome the noise limit set by the corpuscular nature of light, also known as a shot-noise level (SNL). At the SNL, completely uncorrelated particles follow a Poissonian distribution in their numbers. Light with noise properties suppressed below the SNL (squeezed light) plays an essential role in quantum metrology [1], quantum imaging $[2,3]$, and quantum communication $[4,5]$, and can be helpful in bio-sensing [6]. Significant interest in the multi-mode squeezed light stems from the possibility of improving the spatial resolution of optical images $[7,8]$. While single-mode sub-Poissionian light was successfully generated in a wealth of experiments using displaced squeezed vacuum states by means of feedforward [9] and postelection [10,11] techniques, constant-current-driven semiconductor diodes $[12,13]$, conditional preparation of bright multi-mode light with suppressed noise properties has never been demonstrated. The reasons are twofold: (1) the mode mismatch $[14,15]$ in the detection, which always reduces the measured nonclassical correlation between the multi-mode mesoscopic twin beams used for the heralded sub-Poissonian light preparation, and (2) low quantum efficiency and high dark noise of the heralding detectors. While the second problem relates to the quality of equipment, the solution to the first problem is to work with the low-photon number states, for which the excess thermal fluctuations of the unmatched modes are negligible. Therefore, to date, the prepared multi-mode sub-Poissonian light contained at maximum only 12 photons per pulse [16,17]. Because of the low efficiency of the detectors, the achieved suppression of the photon number noise was only $15 \%$ below the SNL. It is a very attractive goal to generate multi-mode mesoscopic nonclassical light that can be used to reduce photon number noise in the applications (for example, in radiometry or quantum imaging) or to probe nonclassical effects in matter by seeding them with bright nonclassical states.

In this Letter, we address both issues listed above. We generate highly multi-mode twin beams containing up to 1.4 photons per mode and $6.3 \cdot 10^{5}$ photons per pulse on the average and detect them separately by two high-efficiency low-noise photodetectors. We observed, to the best of our knowledge, the strongest nonclassical correlations between multi-mode signal and idler squeezed vacuum beams to date. Using the method developed in [10], we conditionally prepare bright multi-mode sub-Poissonian light with the noise suppressed $(37 \pm 1) \%$ below the SNL. Since the pulse contains at least $10^{5}$ photons, it is the strongest source of multi-mode sub-Poissonian nonclassical light. Different from the sub-Poissonian light prepared by displacing the squeezed state, our method does not require the phase stability of the coherent displacement.

The setup is shown in Fig. 1(a). The optical parametric amplifier OPA based on a single type-I $3 \mathrm{~mm}$ thick BBO crystal was pumped by a third harmonic of a Nd:YAG laser at the wavelength $355 \mathrm{~nm}$ with the pulse duration $18 \mathrm{ps}$ and the repetition rate $1 \mathrm{kHz}$. The pump power was changed in the range from 36 to $126.3 \mathrm{~mW}$ by means of a half-wave plate $(\lambda / 2)$ placed in front of a polarization cube (PBS). The diameter of the pump beam (FWHM) at the position of the crystal was $1.5 \mathrm{~mm}$. Signal and idler beams were generated at 635 and $805 \mathrm{~nm}$, respectively. Afterward, the crystal the pump beam was cut off by two dichroic mirrors (DM) with high reflection at $355 \mathrm{~nm}$ and high transmission at 635 and $805 \mathrm{~nm}$, and a colored glass filter OG-630 (OG). The two-color parametric 
(a)

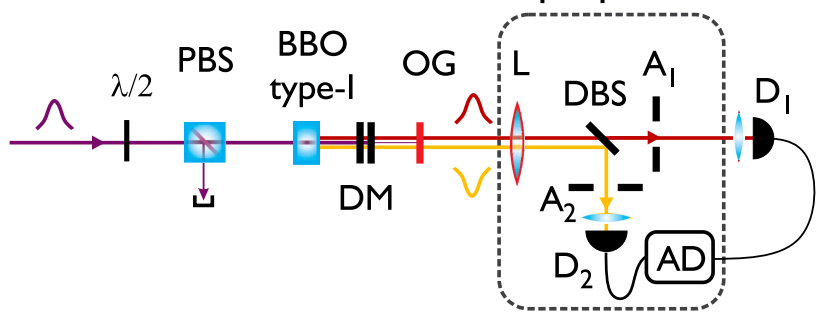

(b) Heralding

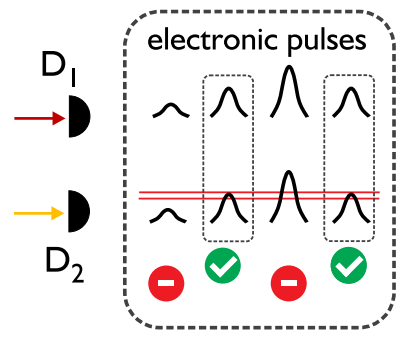

Fig. 1. (a) Experimental setup. (b) Preparation of the subPoissonian light through the heralding of the Channel 2.

beams were separated by a dichroic beamsplitter (DBS). The apertures A1 and A2 of diameters 7 and $8.91 \mathrm{~mm}$, respectively, were inserted into the signal and idler beams in the focal plane of a lens (L) with the focal distance $200 \mathrm{~mm}$. The detected maximal angles satisfied the condition for the conjugate mode detection $\lambda_{s} / \lambda_{i}=\theta_{s} / \theta_{i}$ [14]. After the apertures, all the radiation was focused by two collecting lenses onto detectors $\left(D_{1}\right.$, $D_{2}$ ). The propagation losses from the crystal to the detectors were measured separately and amounted to $7 \%$. According to the datasheet [18], the quantum efficiency (QE) of the PIN diode $\mathrm{S} 3072$ at $635 \mathrm{~nm}$ is $82 \%$, and the QE of the PIN diode $\mathrm{S} 3883$ at $805 \mathrm{~nm}$ is $89 \%$. However, we expect the efficiency to be higher, because the protection glass windows in front of the diodes were removed. The signals from the diodes were amplified and shaped by low-noise charge-sensitive preamplifiers (A250) and shaping amplifiers (A275) by Amptek. Pulses from the detectors were integrated by a 12-bit, eight-channel digitizer NI PXI-5105 by National Instruments with the sample rate of $60 \mathrm{MS} / \mathrm{s}(\mathrm{AD})$. The data were stored in a computer for further processing. The number of photons per mode was estimated from the nonlinear dependence of the PDC signal on the pump power [19]. In the experiment, the mean number of photons per mode $N_{m}$ of the parametric radiation was in the range of 0.3 to 1.4 . For each mean value of the pump power, the data of $3 \cdot 10^{5}$ signal and idler pulses were measured and stored in the computer.

To demonstrate the nonclassical photon-number correlations between the signal and idler beams using the data collected in the measurement, we use the noise reduction factor (NRF), which is given by the formula

$$
\mathrm{NRF} \equiv \frac{\operatorname{Var}\left(N_{1}-k \cdot N_{2}\right)}{\operatorname{Var}\left(N_{1 \mathrm{coh}}-k \cdot N_{2 \mathrm{coh}}\right)},
$$

where $N_{1(2)}$ is the number of detected photons integrated per pulse in the detector $D_{1(2)}$, and $k=\left\langle N_{1}\right\rangle /\left\langle N_{2}\right\rangle$ is a numerical coefficient to compensate for the unbalancing of the detection gains (including detection efficiencies). We equalize the gains of the detectors to compensate for the excess noise caused by the thermal statistics of the detected beams [20]. The value in the denominator is the shot-noise calibration for the given values $N_{1}, N_{2}$ and $k$ measured with the coherent light. If NRF $<1$ the photon number correlation is nonclassical, i.e., it cannot be obtained by a mixture of classical coherent states. Taking into account the electronic noise of the detectors, the formula reads

$$
\mathrm{NRF}_{\text {est }}=\frac{\operatorname{Var}\left(N_{1}-k \cdot N_{2}\right)-\operatorname{Var}\left(V_{D 1}\right)-k^{2} \operatorname{Var}\left(V_{D 2}\right)}{\operatorname{Var}\left(N_{1 \mathrm{coh}}-k \cdot N_{2 \mathrm{coh}}\right)-\operatorname{Var}\left(V_{D 1}\right)-k^{2} \operatorname{Var}\left(V_{D 2}\right)},
$$

where $V_{D 1(2)}$ is the electronic and dark noise of detector $D_{1(2)}$. It should be noted that this equation can only be applied when the electronic noise of the detectors is smaller than the shot-noise level [21]. This was the case in our experiment.

As a first result, we plot NRF calculated according to Eq. (2) against the number of photons per mode in Fig. 2. As expected, we observed a linear dependence [22]. The data were fitted by a linear function according to the formula

$$
\mathrm{NRF}_{\text {est }}=1-\alpha+\beta \cdot N_{m}
$$

where the fitting parameters $\alpha=0.857 \pm 0.004$ and $\beta=0.0916 \pm 0.005$. Both parameters depend on the quantum efficiency of the first detector $\eta_{1}$, ratio of quantum efficiencies of the detectors $k=\frac{\eta_{1}}{\eta_{2}}$, and the number of matched modes $M$ and unmatched ones $K$ as $\alpha=\frac{2 M}{M+K} \frac{\eta_{1}}{1+k}$ and $\beta=\frac{2 K}{M+K} \frac{\eta_{1}}{1+k}$. Assuming that $M \gg K$ and the ratio of quantum efficiencies equals the ratio of the readings of the signal and idler detectors, which can be obtained from the measured data, we obtain $\eta_{1}=(86.2 \pm 0.5) \%$. As one can see from Fig. 2, we observed a reduction of the noise of the photon number difference down to $(16.6 \pm 0.3) \%$ of the SNL for the state containing $N_{m}=0.33$ and $\left\langle N_{1}\right\rangle=1.6 \cdot 10^{5}$. This amounts to $7.8 \mathrm{~dB}$, the largest degree of twin-beam squeezing ever demonstrated

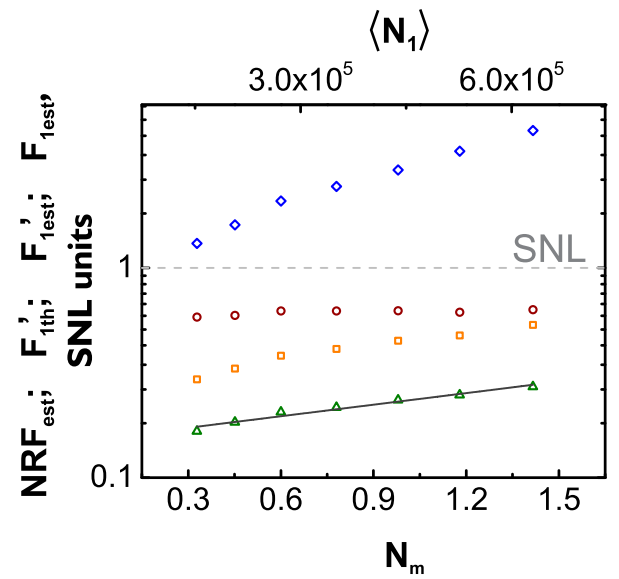

Fig. 2. Experimentally measured noise reduction factor $\mathrm{NRF}_{\text {est }}$ (green triangles), theoretically predicted Fano factor $F_{1 \text { th }}^{\prime}$ (orange squares) according to Eq. (4), the Fano factor $F_{\text {lest }}^{\prime}$ for the heralded state $(Q=20)$ (vinous circles), and the Fano factor for the unconditional state $F_{1 \text { est }}$ (blue diamonds) as functions of the number of photons per mode $N_{m}$ (bottom axis) and mean number of photons per pulse $\left\langle N_{1}\right\rangle$ (top axis). The straight line is a linear fit plotted according to Eq. (3). 
for multi-mode squeezed vacuum states of light, to the best of our knowledge.

Conditional preparation of the sub-Poissonian light is realized using the same data set. The procedure of the data processing is based on post-selection. As shown in Fig. 1(b), for further analysis of detector $D_{1}$ data, we use only those pulses for which the output of detector $D_{2}$ takes values within the range of $\frac{2 \cdot S \mathrm{D}}{Q}$ around the chosen level. Here, SD is the standard deviation of the signal, and $Q$ is a constant defining the conditioning strength. The higher the $Q$, the stronger is the condition.

To quantify the noise of the conditionally prepared light, we calculate the Fano factor $F_{1(2)} \equiv \frac{\operatorname{Var}\left(N_{1(2)}\right)}{\operatorname{Var}\left(N_{1(2) \operatorname{coh}}\right)}=\frac{\operatorname{Var}\left(N_{1(2)}\right)}{\left\langle N_{1(2)}\right)}$. Following [23], in our case, theoretical calculation of $F_{1 \text { th }}^{\prime}$ for the conditionally prepared state in Channel 1 is given by

$$
F_{1 \mathrm{th}}^{\prime}=F_{1}-\frac{\left(F_{1}+F_{2}-2 \mathrm{NRF}\right)^{2}}{4 F_{2}},
$$

where $F_{1,2}$ are the Fano factors for the unconditional beams 1, 2 . Following this, we expect the noise in the prepared beam to be almost twice as large as the noise of the photon number difference.

Taking into account the electronic noise of the detector 1(2), the Fano factor reads

$$
F_{1(2) \text { est }}=\frac{\operatorname{Var}\left(N_{1(2)}\right)-\operatorname{Var}\left(V_{D 1(2)}\right)}{\left\langle N_{1(2)}\right\rangle-\left\langle V_{D 1(2)}\right\rangle} .
$$

Contrary to the NRF calculation (2), in the Fano factor $F_{\text {lest }}^{\prime}$ for the heralded sub-Poissonian light in Channel 1, only the noise of detector $D_{1}$ can be eliminated, but the electronic noise of the control detector (D2) is always present.

We next demonstrate the conditional preparation of the sub-Poissonian light. In Fig. 2, the Fano factor for conditionally prepared states $(Q=20)$ in the target channel (vinous circles) as a function of $N_{m}$ is depicted. The points were calculated according to Eq. (5). The deviation of the experimental results from the theoretical predictions according to Eq. (4) (orange squares) is due to the electronic noise contribution of the control detector (D2). For the brightest state containing $\left\langle N_{1}\right\rangle=6.3 \cdot 10^{5}$ photons per pulse, the Fano factor is found to be $F_{\text {lest }}^{\prime}=0.63 \pm 0.01$, which is slightly larger than $F_{1 \text { th }}^{\prime}=$ $0.53 \pm 0.01$ predicted by Eq. (4) using $\mathrm{NRF}_{\text {est }}=0.314 \pm$ $0.002, F_{1 \text { est }}=4.53 \pm 0.01$, and $F_{\text {2est }}=4.33 \pm 0.01$.

Apart from the electronic noise of the detectors, the signal and the idler channels are influenced by optical losses, finite quantum efficiency, and unmatched modes. Therefore, the selected interval in the control channel should have a finite width. For the brightest conditionally prepared state containing $6.3 \cdot 10^{5}$ photons per pulse, we plot $F_{1 \text { est }}^{\prime}$ as a function of $Q$ in Fig. 3(a). The conditioning was applied around the mean signal in $D_{2}$. As expected, the stronger the condition, the lower is the value of $F_{\text {lest }}^{\prime}$. According to the presented data, the Fano factor reaches the minimum value of $0.63 \pm 0.01$ at $Q=20$ and remains constant for $Q>20$. In general, the stronger the condition, the lower is the success rate of the state preparation. For example, applying the condition of widths SD, SD/4, and $\mathrm{SD} / 10$, we prepare sub-Poissonian light with the Fano factor of $F_{\text {lest }}^{\prime}(1)=0.944 \pm 0.004, F_{\text {lest }}^{\prime}(2)=0.66 \pm 0.01$, and $F_{\text {lest }}^{\prime}(3)=0.63 \pm 0.01$ with the success rate of $40 \%, 10 \%$, and $4 \%$, respectively. The inset presents the probability distributions of the unconditional state (dark gray), the conditionally
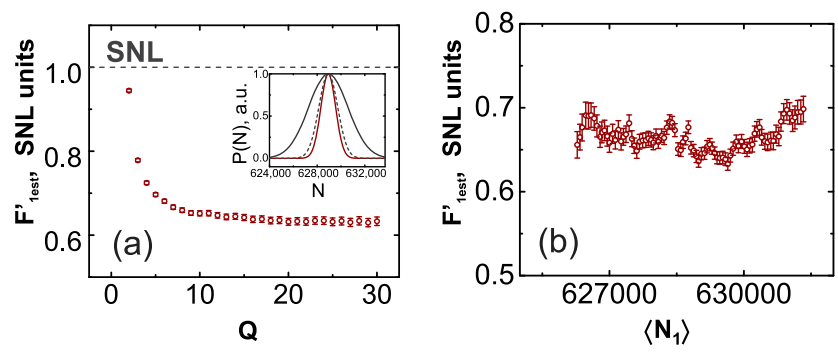

Fig. 3. (a) Fano factor plotted versus the strength $Q$ of the condition for the state containing $6.3 \cdot 10^{5}$ photons per pulse. Inset: probability distribution for the unconditional state (dark gray solid line), shotnoise limited state (light gray dashed line), and a conditionally prepared sub-Poisonian state (vinous solid line). (b) Fano factor versus the mean number of photons $\left\langle N_{1}\right\rangle$ increased by shifting the middle of the conditioning interval with the bandwidth of $\mathrm{SD} / 10(Q=20)$ in Channel 2.

prepared sub-Poissonian state with $Q=20$ (vinous), and the shot-noise limited state (light gray).

To demonstrate the stability of the method, for the target beam we plot $F_{1 \text { est }}^{\prime}$ as a function of the mean number of photons per pulse [Fig. 3(b)]. Each point of the plot was obtained from the same data set, measured at the pump power of $126.3 \mathrm{~mW}$. The selection interval of bandwidth SD/10 $(Q=20)$ was shifted around the mean signal in the control channel. One can see that the mean number of photons in the prepared state increases by $4 \cdot 10^{3}$ photons while the Fano factor remains almost unchanged within the error range.

In conclusion, we have measured a record value of $7.8 \mathrm{~dB}$ twin-beam squeezing for multi-mode two-color squeezed vacuum beams. Sub-Poissonian light containing up to $6.3 \cdot 10^{5}$ photons per pulse and up to 1.4 photons per mode with the Fano factor $F_{\text {lest }}^{\prime}=0.63 \pm 0.01$ has been prepared in the target (signal) beam through the heralding of the control (idler) beam. The obtained pulses of light have the average number of photons from $1.5 \cdot 10^{5}$ to $6.3 \cdot 10^{5}$, corresponding to energies from 0.04 to $0.15 \mathrm{pJ}$, and the energy variance $37 \%$ below the shot-noise level. This makes our technique interesting for radiometry, especially taking into account the possibility to move the idler beam wavelength to the infrared range. Besides, due to the relatively high photon number per mode, the obtained sub-Poissonian beam can be used now to probe the basic quantum interactions in nonlinear optics [24] or quantum optomechanics [25] by mesoscopic nonclassical states of light.

Funding. Seventh Framework Programme (FP7) (308803, 609405); Czech Science Foundation (GB14-36681G, 1327533J).

Acknowledgment. T. Sh. I., V. C. U., R. F., M. V. C., and G. L. acknowledge the financial support of the EU FP7 under grant agreement no. 308803 (project BRISQ2). T. Sh. I. acknowledges the support of the People Programme (Marie Curie Actions) of the European Union's Seventh Framework Programme (FP7/2007-2013) under REA grant agreement no. 609405 (COFUNDPostdocDTU). R. F. acknowledges project GB14-36681G of the Czech Science Foundation. V. C. U. acknowledges project 13-27533J of the Czech Science Foundation. 


\section{REFERENCES}

1. G. Brida, M. Chekhova, M. Genovese, and I. Ruo-Berchera, Opt. Express 16, 12550 (2008).

2. G. Brida, M. Genovese, and I. Ruo Berchera, Nat. Photonics 4, 227 (2010).

3. M. Genovese, "Real applications of quantum imaging," arXiv:1601. 06066v1 (2016).

4. M. Nielsen and I. Chuang, Quantum Computation and Quantum Information (Cambridge University, 2000).

5. L. S. Madsen, V. C. Usenko, M. Lassen, R. Filip, and U. L. Andersen, Nat. Commun. 3, 1083 (2012).

6. M. A. Taylor, J. Janousek, V. Daria, J. Knittel, B. Hage, H.-A. Bachor, and W. P. Bowen, Nat. Photonics 7, 229 (2013).

7. M. I. Kolobov, Rev. Mod. Phys. 71, 1539 (1999).

8. M. I. Kolobov and C. Fabre, Phys. Rev. Lett. 85, 3789 (2000).

9. J. Mertz, A. Heidmann, C. Fabre, E. Giacobino, and S. Reynaud, Phys. Rev. Lett. 64, 2897 (1990).

10. J. Laurat, T. Coudreau, N. Treps, A. Maître, and C. Fabre, Phys. Rev. Lett. 91, 213601 (2003).

11. H. Zou, S. Zhai, J. Guo, R. Yang, and J. Gao, Opt. Lett. 31, 1735 (2006) and the references within it.

12. P. R. Tapster, J. G. Rarity, and J. S. Satchel, Europhys. Lett. 4, 293 (1987).

13. S. Machida and Y. Yamamoto, Phys. Rev. Lett. 60, 792 (1988).
14. I. N. Agafonov, M. V. Chekhova, and G. Leuchs, Phys. Rev. A 82, 011801(R) (2010).

15. M. A. Finger, T. Sh. Iskhakov, N. Y. Joly, M. V. Chekhova, and P. St.J. Russell, Phys. Rev. Lett. 115, 143602 (2015).

16. J. Peřina, Jr., O. Haderka, and V. Michálek, Opt. Express 21, 19387 (2013).

17. M. Lamperti, A. Allevi, M. Bondani, R. Machulka, V. Michlek, O. Haderka, and J. Perjina, Jr., J. Opt. Soc. Am. B 31, 20 (2014).

18. Selection guide by Hamamatsu http://www.hamamatsu.com/ resources/pdf/ssd/sipdkspd0001e.pdf.

19. O. A. Ivanova, T. Sh. Iskhakov, A. N. Penin, and M. V. Chekhova, Quantum Electron. 36, 951 (2006).

20. G. Brida, I. P. Degiovanni, M. Genovese, M. L. Rastello, and I. Ruo-Berchera, Opt. Express 18, 20572 (2010).

21. I. Agafonov, M. Chekhova, T. Iskhakov, and G. Leuchs, Quant. Cryptogr. Comput. 26, 161 (2010).

22. T. Sh. Iskhakov, V. C. Usenko, R. Filip, M. V. Chekhova, and G. Leuchs, "Low-noise macroscopic twin beams," arXiv:1408.6407v1 (2014).

23. J. Laurat, T. Coudreau, N. Treps, A. Maître, and C. Fabre, Phys. Rev. A 69, 033808 (2004).

24. F. De Martini, F. Sciarrino, and C. Vitelli, Phys. Rev. Lett. 100, 253601 (2008).

25. M. R. Vanner, J. Hofer, G. D. Cole, and M. Aspelmeyer, Nat. Commun. 4, 2295 (2013). 